\title{
Evaluation of Drug-Related Problems in a Pediatric Bone Marrow Transplantation Unit Identified by a Clinical Pharmacist in-training in a 7-Month Period
}

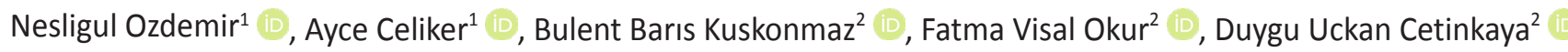 \\ ${ }^{1}$ Hacettepe University Faculty of Pharmacy, Department of Clinical Pharmacy, Ankara, Turkey \\ ${ }^{2}$ Hacettepe University Faculty of Medicine, Department of Pediatric Hematology, Ankara, Turkey
}

Correspondence Author: Nesligul Ozdemir

E-mail: nesliozdmr@hotmail.com

Received: $10.07 .2019 \quad$ Accepted: 22.11.2019

\begin{abstract}
Objective: This cross sectional study was performed to demonstrate the importance of clinical pharmacists' participation in pediatric hematopoietic stem cell transplantation patient management with regards to the detection, prevention, and management of drug-related problems.

Methods: The study was carried out from 1st October 2015 to 1st May 2016 in a pediatric bone marrow transplantation unit of a tertiary care hospital. The inpatients and outpatients between 0 to 18 years of age were included and the patients were monitored and evaluated for drugrelated problems (interactions, side effects, preparation, and administration). A clinical pharmacist-in-training made recommendations to the physicians and the nurses on the problems that were identified.

Results: Twenty inpatients and twenty-two outpatients were monitored during the study. In total, 245 problems were identified in the inpatients, $37.14 \%$ of which were drug-related; $33 \%$ of the drug-related problems were the side effects of cyclosporine. Eleven recommendations on drug-related problems were made to the physicians and six of them were for the problems identified in the inpatient services with regards to drug dosing and administration. Five recommendations regarding total parenteral nutrition, drug incompatibility, drug administration from the feeding tube, and drug side effects were made to the nurses. Twenty-nine information on the dosing, side effects, incompatibilities, administration, and preparation of the drugs, were given by the pharmacist to the physicians and the nurses.

Conclusion: Clinical pharmacists' participation will improve the detection and the management of drug-related problems in pediatric hematopoietic stem cell transplantation units in Turkey.
\end{abstract}

\section{INTRODUCTION}

Hematopoietic Stem Cell Transplantation (HSCT) is currently a common medical practice in the world; it is performed on > 40,000 patients annually as reported in European Blood and Marrow Transplantation Registry (1). HSCT procedure involves several stages consisting of the administration of highly toxic preparative regimens (chemotherapy and/or radiotherapy), intravenous (IV) infusion of hematopoietic stem cells, administration of prophylactic and supportive medications for the prevention of side effects, and administration of medications for the treatment/management of complications (2-4). The use of polypharmacy for prophylaxis and treatment may lead to adverse effects and drug interactions $(5,6)$. Because of the pharmacokinetic differences in the childhood and adulthood stages, it is necessary to be more careful in monitoring adverse and side effects in pediatrics (7).

The exponential increase in HSCT activities has led to the inclusion of pharmacists in HSCT practice throughout the world. Clinical pharmacists have become critical members of HSCT teams for the last 10 years (6). The presence of a clinical pharmacist in the HSCT team is an approach aimed at improving the quality of care, through their contribution to detection, prevention and management of drug-related problems (DRPs) $(8,9)$. The involvement of a dedicated pharmacist is particularly important in pediatric HSCT practice because children are highly prone to the side effects of drugs due to pharmacokinetics reasons (10). Many transplantation units in developed countries have pharmacy staff in their multidisciplinary teams who routinely attend clinical rounds and are actively involved in the management of DRPs and in decision making. However, this has not become a standard practice in most of the units in the developing countries including Turkey. The involvement of a clinical pharmacist in a HSCT team has become a requirement in the recent editions of the Joint Accreditation Committee of International Society for Cellular Therapy-European Group for Blood and Marrow Transplantation/The Foundation for the Accreditation of Cellular Therapy standards, and this is expected to play a role in generalizing this practice for all HSCT Units (11). 
HSCT activities have risen sharply in Turkey since 2010 and approximately 3500 transplantations are performed annually with one-fourth of those in children (12). In pediatric practice, due to the heterogeneity of diagnoses, transplantation practice necessitates an age and diagnosis specific approach in the management of patients, including the choice and the administration of medications. Therefore, there is a need for the active involvement of pharmacists in pediatric practice, particularly in centers performing transplantation in complicated patients with primary immunodeficiency, inborn errors of metabolism, or systemic problems.

The present pilot study was performed to investigate the role of a clinical pharmacist-in-training in the detection and the identification of DRPs in pediatric HSCT patients in a given period, and to assess the impact of this practice on patient management, with regards to the sharing of information by the clinical team members.

\section{METHODS}

This study was carried out from 1st October 2015 and 1st May 2016 in a tertiary care hospital's pediatric bone marrow transplantation unit, as a prospective observational study. A written informed consent was obtained from the patients or their parents and the study was ethically approved by the Hacettepe University Ethics Committee of Non-Interventional Clinical Investigations (GO 15 / 596-04, dated 21.10.2015). Inpatients and outpatients between 0 to 18 years of age were included in the study. Patients who did not want to take part in the study and those who had a short-term hospitalization period of drug administration were not included in the study.

The pharmacist was a graduate student (Masters) in the clinical pharmacy program. The treatment of the inpatients was prospectively monitored. The pharmacist attended the physicians' visits in the inpatient service between 9 am and 12 am, 4 days a week. The outpatients, using at least one drug, were followed up with face-to-face interviews conducted by the pharmacist once a week. Patient information was collected daily, through chart review.

The assumed DRPs were identified, and the side effects were observed. Pharmaceutical Care Network Europe defines a drug related problem as "an event or circumstance involving drug therapy that actually or potentially interferes with desired health outcomes". Drug related problems in this study were identified according to above mentioned definition. To distinguish disease and drug related side effect, suspected drug was stopped or the dose was decreased and the given symptom was evaluated again. If the symptom disappeared or was relieved, it was accepted as drug related. Drug orders were evaluated with regards to supportive therapy doses, the duration of treatment, and drug interactions in accordance with current guidelines and publications. The blood levels of narrow therapeutic index drugs were monitored and evaluated. The recommendations of the pharmacist with regards to dose adjustment, drug interactions, and the alteration of drug administration times were negotiated verbally with the physicians. For the evaluation of side effects, pharmacokinetic information and drug interactions, Micromedex Solutions ${ }^{\circledR}$, Medscape ${ }^{\circledR}$ and UpToDate ${ }^{\circledR}$ online sources were used.

IBM SPSS version 23 program was used for the statistical analysis of the data in the study. The distribution of the data was calculated through descriptive statistics.

\section{RESULTS}

During the study period, the pharmacist monitored 20 inpatients and 22 outpatients (median ages $=8$, ranges $=0.6$ 17 and 2-17 years, respectively). All the patients received transplants from family donors, except one autologous. A total of 177 patients visited the HSCT outpatient clinic and only 56 of them were receiving more than one medication in the study period. These patients visited the outpatient clinic at least once after HSCT. The pharmacist interviewed only 22 outpatients (10 of them were inpatients, and they were included after they were discharged once during the study period).

The demographic data of the patients are summarized in Table 1. In the majority of the inpatients, the primary diseases were acute lymphoblastic leukemia (ALL), thalassemia major and Fanconi anemia; and in the outpatients, the primary diseases were thalassemia major, ALL and Wiskott-Aldrich syndrome, respectively.

Table 1. Demographic data of inpatients and outpatients

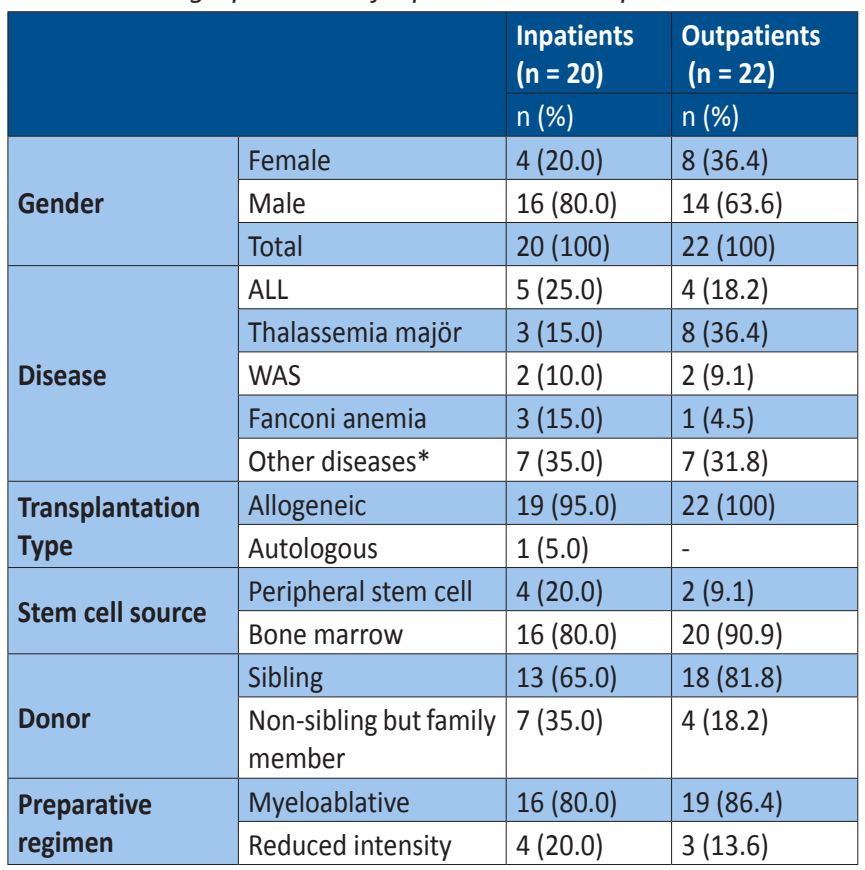

ALL: Acute lymphoblastic leukemia, WAS: Wiskott Aldrich Syndrome. Sibling donor means patient's sister or brother, Non-sibling but family members (haploidentical): mother, father, aunt, and cousin. Other diseases*: there was one inpatient for each disease-osteopetrosis, neuroblastoma, congenital neutropenia, adrenoleukodistrophy, lipopolysaccharideresponsive beige-like anchor gene defect-immunodeficiency, and juvenile myelomonocytic leukemia. There was one outpatient for each diseaseosteopetrosis, immunodeficiency due to osteopetrosis, adenosine deaminase enzyme deficiency, aplastic anemia, acute myeloid leukemia, congenital neutropenia, and myelodysplastic syndrome 
A total of 245 problems were encountered in the inpatients. The most common problems were; low-grade fever (fever between $37.5^{\circ} \mathrm{C}$ and $38.3^{\circ} \mathrm{C}$ ), neutropenic fever, nausea, vomiting, and diarrhea, which were observed in nearly all the patients. The problems are summarized in Table 2 . Of the problems, 91 were DRPs. The DRPs are summarized in Table 3. The majority ( $33 \%$ ) of the side effects was associated with cyclosporine. Furthermore, most of the detected side effects could not have been prevented because they were associated with essential HSCT drugs which could not have been withdrawn. Cyclosporine, methotrexate, and caspofunginrelated side effects such as hypertension, dyslipidemia, and hyperglycemia were managed by the adjustment of drug doses. After the detection of the side effects related to the above mentioned drugs by the pharmacist, the physicians were informed and the necessary adjustments on drug dosages were made with the consensus of the pharmacist and the physicians.

Table 2. Distribution of problems.

\begin{tabular}{|l|c|c|}
\hline Problems & Number (n) & Percentage (\%) \\
\hline Nausea & 15 & 6.1 \\
\hline Vomiting & 13 & 5.3 \\
\hline Diarrhea & 13 & 5.3 \\
\hline Low grade fever & 11 & 4.5 \\
\hline Neutropenic fever & 8 & 3.3 \\
\hline Increase in CMV copy & 8 & 3.3 \\
\hline Chronic GVHD & 8 & 3.3 \\
\hline Cytopenia & 5 & 2.0 \\
\hline Others & 73 & 29.8 \\
\hline Drug-related problems & 91 & 37.1 \\
\hline Total & 245 & 100 \\
\hline
\end{tabular}

CMV: Cytomegalovirus, GVHD: Graft versus Host Disease. Others: Acute GVHD, bacterial catheter colonization, Engraftment Syndrome, hypotension, tachycardia, bradycardia, hypothyroidis, electrolyte imbalance, catheter infection, convulsion, pancreatitis, cellulite, cough, weight loss, stomachache, epigastric pain, headache, hyperemia, bacterial colonization in urine culture, CMV retinitis, hypotermia, refractory thrombocytopenia, dysphagia, hemolysis, gastrointestinal bleeding, cortical blindness, respiratory arrest, angioedema, feeding problem.

Sixteen consultations were given to the physicians. They included those on side effects (25\%), dosing and administration (25\%), drug blood levels (12.5\%) and others (12.5\%).

In total, six recommendations were made to the physicians for the problems identified in the inpatients with regards to drug dosing and administration. One recommendation was about changing the dosage schedule of interacting medicines, three recommendations were about reducing the dose, one recommendation was about converting 'thrice daily dosing' of amikacin to 'single daily dosing', and one recommendation was about switching to another formulation. Except one, which was about dose reduction, all the recommendations were accepted by the physicians.
Table 3. Distribution of drug-related problems.

\begin{tabular}{|l|c|c|}
\hline Problems & Number (n) & Percentage (\%) \\
\hline $\begin{array}{l}\text { Preparative regimen related mucositis } \\
\text { (stage 1-2-3-4) }\end{array}$ & 43 & 47.2 \\
\hline $\begin{array}{l}\text { Cyclosporine induced triglyceride and } \\
\text { cholesterol levels elevation }\end{array}$ & 7 & 7.7 \\
\hline $\begin{array}{l}\text { Chemotherapy or cyclosporine induced } \\
\text { hypertension }\end{array}$ & 18 & 19.8 \\
\hline \begin{tabular}{l} 
Steroid induced hyperglycaemia \\
\hline $\begin{array}{l}\text { Cyclosporine-caspofungin interaction } \\
\text { induced increase in liver transaminases }\end{array}$
\end{tabular} & 3 & 3.4 \\
\hline $\begin{array}{l}\text { Methotrexate induced increase in liver } \\
\text { transaminases }\end{array}$ & 2 & 2.2 \\
\hline $\begin{array}{l}\text { Cyclosporine induced posterior reversible } \\
\text { encephalopathy syndrome }\end{array}$ & 2 & 2.2 \\
\hline $\begin{array}{l}\text { Intravenous immunoglobulin related fever } \\
\text { and rash }\end{array}$ & 2 & 2.2 \\
\hline Others & 10 & 11.0 \\
\hline Total & 91 & 100 \\
\hline
\end{tabular}

Others: There was one patient for each problem; renal dysfunction after foscarnet administration, etoposide related hyponatremia, etoposide related hypokalemia, fever after Anti-thymocyte globulin administration, high dose administration of tacrolimus, foscarnet related nausea, foscarnet related vomiting, azacitidine related diarrhea, azacitidine related weight loss and regimen related hemorrhagic cystitis.

Five recommendations were made with regards to the outpatients; two of them were about dissolving the drug in apple juice to aid swallowing, two were about changing the administration times of interacting drugs, and the last recommendation was about addition of a new drug for an untreated indication. Except the addition of a new drug, all the recommendations were accepted by the physicians. At outpatients, the problems observed directly by pharmacist was evaluated so the other problems such as diarrhea, vomiting, etc. could not be assessed in the limited interview time.

A total of five recommendations were made to the nurses. Two of the recommendations were about the preparation of the drugs, one was about preventing the precipitation in the total parenteral nutrition (TPN) tract, one was about drug administration through an enteral feeding tube, and the last recommendation was about the prevention of side effects. The total 13 consultations given to the nurses were about drug-drug incompatibilities (46.2\%), drug preparation and administration (30.7\%), and TPN-drug incompatibility and drug administration time (23.1\%). Necessary actions were taken by the nurses.

\section{DISCUSSION}

In the literature, there are several studies showing clinical pharmacists' impact, especially on the detection of medication errors, prevention of drug-drug interactions and development of supportive treatment for adverse reactions in pediatric HSCT units. Nevertheless, in Turkey, including the department where this study was conducted, clinical pharmacists are not routinely involved in these activities. 
In the present study, we investigated the role of a clinical pharmacist-in training in the detection and the prevention of DRPs in pediatric HSCT patients in Turkey for the first time. We evaluated the complications encountered during the transplantation period and the problems caused by the drugs. Furthermore, we recorded the outcomes of the recommendations of the pharmacist on the HSCT activities. Before the clinical pharmacist's participation, the whole process of the HSCT was being monitored by only the physicians and the nurses, wasting their limited resources and time for DRPs.

The most common DRPs identified were mucositis, dyslipidemia, hypertension, and hyperglycemia.

Oral mucositis develops in $75-85 \%$ of patients who undergo a myeloablative preparation regimen $(13,14)$. In a metaanalysis, $79 \%$ of the patients receiving the myeloablative regimen and $71 \%$ of the patients receiving non-myeloablative regimens had severe mucositis (grade $2,3,4$ ) according to the World Health Organization and the National Cancer Institute (NCl) toxicity criteria (15). In our study, all the patients had grade 1 mucositis (according to $\mathrm{NCl}$-Common Toxicity Criteria version 2.0). Grade 4 mucositis (mucositis requiring TPN) was seen in $30 \%$ of 20 patients. In our study, the rate of grade 1 mucositis was similar to those of other studies; however, the rate of severe mucositis was lower than that of other studies owing to the effective preventive and supportive treatments for mucositis in our unit.

Dyslipidemia is a late-onset post-transplantation problem associated with factors such as obesity, genetic lipid disorders, primary disease, transplantation, and its complications, TPN, use of cyclosporine, chronic graft versus host disease (GVHD), and individual condition and other diseases. Dyslipidemia observed in the early post-transplantation period is associated with calcineurin inhibitors $(16,17)$. In a study conducted by Mehdizadeh et al. (18), $95 \%$ of adult patients using cyclosporine had dyslipidemia. In our study, we found that $35 \%$ of the patients on cyclosporine developed dyslipidemia. There was a decrease in the basal levels of triglyceride and cholesterol when the dose of cyclosporine was reduced; hence, we inferred that dyslipidemia was associated with cyclosporine in our patients.

Hypertension, a result of preparative regimens and immunosuppresants, is frequently observed in pediatric patients. It has been demonstrated that cyclosporine, one of the causes of hypertension, significantly increases blood pressure in studies in comparison with placebo (19). In Kishi and his colleagues' study (20), cyclosporine dose needed to be reduced in $64 \%$ of adult patients because of the incidence of hypertension. In the present study, almost all the patients had elevated blood pressure $(>140 / 90 \mathrm{mmHg})$ at least once during the study. The exact cause of hypertension has not been clearly elicited, but it has been considered as a side effect of calcineurin inhibitors and preparative regimens including total body irradiation. In this study, unlike the other studies, cyclosporine dose was not modified, but hypertension was managed with antihypertensive treatment.
Calcineurin inhibitors and corticosteroids are important drugs used in the prophylaxis and treatment of GVHD through immunosuppression. However, as a result of long-term use of steroids, side effects such as hyperglycemia and prolonged hospitalization are observed in patients (21). In Fuji and colleagues' study (22), $64 \%$ of patients' fasting blood glucose values were higher than $126 \mathrm{mg} / \mathrm{dl}$. In the present study, 14 patients (70 \%) had a higher fasting blood glucose level than $126 \mathrm{mg} / \mathrm{dl}$, and hyperglycemia (> $200 \mathrm{mg} / \mathrm{dl}$ ) was observed in four patients due to a long-term use of steroid. The results showed that steroid and calcineurin inhibitors should be used carefully, and patients' blood glucose levels should be monitored regularly in terms of the other factors that might influence the blood glucose levels, such as TPN.

The studies carried out in more developed HSCT units showed that collaborations between pharmacists and physicians increased the quality and efficiency of the patients' care. The establishment of a system, in which physicians and pharmacists negoitate on DRPs, will positively affect the health outcomes of the patients and also alleviate the workload of the physicians (23). In our study, DRPs were generally known as the side effects of drugs, and they were checked by considering the patient's individual response in the treatment process. Nonetheless, the adverse effects occurred due to the essential drugs (immunosuppressants) for HSCT, and these drugs could not be withdrawn. Compared to the in this study the number of recommendations made by the pharmacist for the detected errors was very few. Most of the recommendations were about the regulation of drug administration times of interacting drugs. A good documentation of the HSCT treatment process in our unit such as the lists of the calculated doses of the drugs per kilogram for each patient, contributed to the reduction of medication errors. Also, the presence of experienced physicians and nurses in the team had a positive impact on the treatment outcomes.

In the outpatients as well as in the inpatients, the regulation of the administration times of different drugs causing pharmacokinetic interactions when given at the same time, and suggestions such as the mixing of drugs with apple juice for patients who had difficulty in swallowing were the interventions made by the pharmacist.

In one of the studies conducted in the pediatric HSCT units, clinical pharmacists' involvement had significant contribution to the development of the protocols for the prevention of adverse effects and drug interactions through the adjustment of drug doses and the selection of appropriate supportive treatments (such as pain management and mucositis prevention) for complications (6). During the seven-month period of our study, consultancy on different issues was provided by the pharmacist to the physicians. The majority of the consultations were about side effects, dose adjustment, and information on unexpected symptoms in the patients. The rates of consultation and intervention on drug dose adjustment and drug side effects in this study were similar to those in the literature (24-26). 
Drug administration errors are common problems (19-27 \%) and have negative consequences for patients. Particularly, the preparation and the administration of IV drugs involve more than one step, thus, giving room for mistakes (27). Ghaleb et al. (28) reported that, errors in drug administration and the wrong infusion of IV drugs were the most common problems in pediatric patients. In this study, when the pharmacist's consultations with the nurses were evaluated, we found that the majority of the problems originated from drug incompatibilities and difficulties in drug preparation processes. In our study, nurses needed pharmacist's support mostly in the same fields as reported in the literature. One of the recommendations made was the preparation of cyclosporine infusion not only with dextrose, but also with saline. This suggestion hastened the preparation of cyclosporine infusion.

\section{CONCLUSION}

This study, being the first to evaluate a clinical pharmacist's role in the pediatric HSCT unit in Turkey, can be a guide for other HSCT units in our country. However, the seven-month study period, and the small number of participating patients were considered as limiting factors. In order to demonstrate a clinical pharmacist's role more effectively, a larger number of patients will be required.

The administration of cytotoxic drugs at high doses, a high probability of complications, and the use of a large number of high-cost drugs necessitate the care of a multidisciplinary team during the long and complex process of HSCT therapy. As HSCT team members, clinical pharmacists can evaluate all clinical findings related to drugs, they can check doses, identify drug interactions, prevent side effects, and provide recommendation for drug preparation and administration. Therefore, the support of a clinical pharmacist to physicians and nurses will help to prevent possible DRPs, and generally improve the success of HSCT.

\section{Acknowledgements}

The authors would like to thank Nevin Cetin (Nurse Coordinator of Pediatric Bone Marrow Transplantation Unit, Hacettepe University Ihsan Dogramaci Children's Hospital) and Fatma Kırac (Head Nurse) for supporting clinical activities, and Konca Yılmaz (Outpatient Clinic Secretary) and Guliz Karatas (Outpatient Clinic Nurse) for their support on the collection of patients' data.

\section{REFERENCES}

[1] European Society for Blood and Marrow Transplantation, Annual Report 2017. 2018. Available from; https://www.ebmt. org/ebmt/documents/ebmt-annual-report-2017. Accessed 2 Jan 2019.

[2] Hatzimichael E, Tuthill M. Hematopoietic stem cell transplantation. Stem Cells Cloning 2010;3:105-117.
[3] Yeşilipek MA. Hematopoetic stem cell transplantation in children. Turk Pediatri Ars 2014;49:91-18.

[4] Applebaum FR. The current status of hematopoietic cell transplantation. Annu Rev Med 2003;54:491-512

[5] Ghaleb MA, Barber N, Franklin BD, Yeung VWS, Khaki ZF, Wong IC. Systematic review of medication errors in pediatric patients. The Annals of Pharmacotherapy 2006;40:1766-1776.

[6] Prot-Labarthe $S$, Therrien $R$, Demanche $C$, Larocque $D$, Bussières JF. Pharmaceutical care in an inpatient pediatric hematopoietic stem cell transplant service. J Oncol Pharm Pract 2008;14:147-152.

[7] Lu H, Rosenbaum S. Developmental pharmacokinetics in pediatric populations. J Pediatr Pharmacol Ther 2014;19:262276.

[8] Barfield RC, Kasow KA, Hale GA. Advances in pediatric hematopoietic stem cell transplantation. Cancer Biol Ther 2008;7:1533-1539.

[9] Ratko TA, Belinson SE, Brown HM, Noorani HZ, Chopra RD, Marbella A, Samson DJ, Bonnell CJ, Ziegler KM, Aronson N. Hematopoietic stem-cell transplantation in the pediatric population. Rockville (MD):Agency for Healthcare Research and Quality 2012. Report no: 12-EHC018-EF

[10] Ferranti J, Horvath MM, Cozart H, Whitehurst J, Eckstrand J. Reevaluating the safety profile of pediatrics: a comparison of computerized adverse drug event surveillance and voluntary reporting in the pediatric environment. Pediatrics. 2008 May doi: 10.1542/peds.2007.2609. [Epub ahead of print].

[11] Hematopoietic Cellular Therapy Accreditation Manual, 7th edition, JACIE/FACT. 2018.Available from; https://www.ebmt. org/jacie-standards/7th-edition-effective-june-1st-2018. Accessed 2 Jan, 2019.

[12] Turkey Stem Cell Coordination Center (TURKOK), Statistics. 2018. Available from; https://turkok.saglik.gov.tr/TR,21122/ istatistikler.html . Accessed 2 Jan, 2019.

[13] Glotzbecker B, Duncan C, Alyea E, Campbell B, Soiffer R. Important drug interactions in hematopoietic stem cell transplantation: what every physician should know. Biol Blood Marrow Transplant 2012;18:989-1006.

[14] Deeg HJ. Optimization of transplant regimens for patients with myelodysplastic syndrome (MDS). Hematol Am Soc Hemat 2005;167-173.

[15] Chaudhry HM, Bruce AJ, Wolf RC, Litzow MR, Hogan WJ, Patnaik MS, Kremers WK, Phillips GL, Hashmi SK. The incidence and severity of oral mucositis among allogeneic hematopoietic stem cell transplantation patients: a systematic review. Biol Blood Marrow Transplant 2016;22:605-616.

[16] Griffith ML, Savani BN, Boord JB. Dyslipidemia after allogeneic hematopoietic stem cell transplantation: evaluation and management. Blood 2010;116:1197-1204.

[17] Törlén J, Ringdén $O$, Garming-Legert $K$, Ljungman $P$, Winiarski J, Remes K, Remes MI, Remberger M, Mattsson J. A prospective randomized trial comparing cyclosporine/methotrexate and tacrolimus/sirolimus as graft-versus-host disease prophylaxis after allogeneic hematopoietic stem cell transplantation. Haematologica 2016;101:1417-1425.

[18] Mehdizadeh M, Hajifathali A, Tafazoli A. Drug utilization evaluation of cyclosporine in allogeneic hematopoietic stem cell transplantation. Exp Clin Transplant 2015;13:461-466.

[19] Robert N, Wong GW, Wright JM. Effect of cyclosporine on blood pressure. Cochrane Database Syst Rev 2010; https://doi: 10.1002/14651858 
[20] Kishi Y, Murashige N, Kami M, Miyakoshi S, Shibagaki Y, Hamaki T, Takaue $Y$, Taniguchi S. Optimal initial dose of oral cyclosporine in relation to its toxicities for graft-versus-host disease prophylaxis following reduced-intensity stem cell transplantation in Japanese patients. Bone Marrow Transplant 2005;35:1079-1082.

[21] Jenssen T, Hartmann A. Emerging treatments for posttransplantation diabetes mellitus. Nat Rev Nephrol 2015;11:465-477.

[22] Fuji S, Rovó A, Ohashi K, Griffith M, Einsele H, Kapp M, Mohty M, Majhail NS, Engelhardt BG, Tichelli A, Savani BN. How do I manage hyperglycemia/post-transplant diabetes mellitus after allogeneic HSCT. Bone Marrow Transplant 2016;51:10411049.

[23] Komanduri KV. Pharmacists and physicians in hematopoietic stem cell transplantation: advances and opportunities to use collaborative practice agreements to improve care. Biol Blood Marrow Transplant 2013;19:505-508.
[24] Ho L, Akada K, Messner H, Kuruvilla J, Wright J, Seki JT. Pharmacist's role in improving medication safety for patients in an allogeneic hematopoietic cell transplant ambulatory clinic. Can J Hosp Pharm 2013;66:110-117.

[25] Kelly DV, Bishop L, Young S, Hawboldt J, Phillips L, Keough TM. Pharmacist and physician views on collaborative practice: Findings from the community pharmaceutical care project. Can Pharm J (Ott) 2013;146:218-226.

[26] Berhane A, Ali E, Odegard P, Suleman S. Physicians' expectations of clinical pharmacists' roles in Jimma University Specialized Hospital, South west Ethiopia. Int J Pharm Teach Pract 2013;4:571-574.

[27] Westbrook JI, Rob MI, Woods A, Parry D. Errors in the administration of intravenous medications in hospital and the role of correct procedures and nurse experience. BMJ Qual Saf 2011;20:1027-1034.

[28] Ghaleb MA, Barber N, Franklin BD, Wong IC. The incidence and nature of prescribing and medication administration errors in paediatric inpatients. Arch Dis Child 2010;95:113-118. 